\title{
Transformation der Siedlungswasserwirtschaft - Steuerungsmechanismen im Diskurs ressourcenorientierter Systemansätze am Beispiel von Thüringen
}

\section{Transformation of the wastewater sector - The ability of state level controlling mechanisms to enhance the implementation of resource-oriented sanitation systems}

https://doi.org/10.2478/rara-2020-0012

Eingegangen: 17. Juni 2019; Angenommen: 4. März 2020

Kurzfassung: Neuartige Sanitärsysteme zielen auf eine ressourcenorientierte Verwertung von Abwasser ab. Erreicht werden soll dies durch die separate Erfassung von Abwasserteilströmen. In den Fachöffentlichkeiten der Wasserwirtschaft und Raumplanung werden neuartige Sanitärsysteme als ein geeigneter Ansatz für die zukünftige Sicherung der Abwasserentsorgung in ländlichen Räumen betrachtet. Die Praxistauglichkeit dieser Systeme wurde zwar in Forschungsprojekten nachgewiesen, bisher erschweren jedoch für Abwasserentsorger vielfältige Risiken die Einführung einer ressourcenorientierten Abwasserbewirtschaftung. Ausgehend von einer Untersuchung der Kontexte bei der Umsetzung eines neuartigen Sanitärsystems im ländlichen Raum Thüringens wird in diesem Beitrag der Frage nachgegangen, wie auf Landesebene mit dem abwasserwirtschaftlichen Instrumentarium die Einführung von ressourcenorientierten Systemansätzen unterstützt werden kann. Zentrale Elemente des Beitrags sind die Darstellung der wesentlichen Transformationsrisiken in Bezug auf die Einführung innovativer Lösungsansätze, eine Erläuterung der spezifischen abwasserwirtschaftlichen Instrumente sowie die Darlegung von Steuerungsansätzen, mit denen die Einführung von neuartigen Sanitärsystemen gefördert werden kann. Im Ergebnis wird die Realisierbarkeit von neuartigen Sanitärsystemen durch den strategischen Einsatz des Instrumentariums deutlich, gleichwohl die Wasserwirtschaft durch die Erweiterung der bisherigen Systemgrenzen auf die Kooperation mit anderen Bereichen der Daseinsvorsorge angewiesen ist.

Schlüsselwörter: Raumplanung, Abwasserwirtschaft, neuartige Sanitärsysteme, Instrumente, Steuerungsansätze, Transformationsrisiken

\footnotetext{
*Corresponding author: Mario Wolf, Bauhaus-Universität Weimar, Bauhaus-Institut für zukunftsweisende Infrastruktursysteme, Professur Siedlungswasserwirtschaft, Coudraystraße 7, 99423 Weimar, Deutschland, E-Mail: ulrich.mario.wolf@uni-weimar.de; ORCID: 0000-0002-0972-1117

Prof. Dr.-Ing. Jörg Londong, Bauhaus-Universität Weimar, Bauhaus-Institut für zukunftsweisende Infrastruktursysteme, Professur Siedlungswasserwirtschaft, Coudraystraße 7, 99423 Weimar, Deutschland
} 
Abstract: New sanitary systems are an intelligent way to approach wastewater management in the face of demographic and climatic changes. It is also compatible with the emerging paradigm of a resource-oriented management of wastewater. While the general technical applicability of resource-oriented systems has been proven in various projects, the realisation is still on hold. The reasons can be found in several risks for wastewater disposal companies that are linked to the implementation process. Based on an analysis of the general context of an implementation of a new sanitary system in a typical rural area of Eastern Germany, this paper analyses to which extend the implementation of such innovative approaches can be facilitated by the regulation system and which steps need to be taken. According to this aim, risks that can hamper the transformation of the wastewater sector are identified, major administrative controlling mechanisms outlined and depicted in which strategic approach these could be used in order to foster the implementation of resource-oriented sanitary systems. As a result, the feasibility of the implementation of new alternative sanitary systems through the strategic application of the controlling mechanisms is generally proven. However, collaborations of the wastewater sectors with stakeholders of other sectors are required.

Keywords: Urban and regional planning, Wastewater management, New sanitary systems, Instruments, Management approaches, Transformation risks

\section{Einführung}

In den vergangenen Jahren haben vielfältige räumliche und gesellschaftliche Veränderungsprozesse die Sicherung der Daseinsvorsorge zunehmend erschwert. Entstandene regionale Disparitäten fordern damit oftmals neue und integrative Handlungsansätze (vgl. ARL 2016). Als Teilbereich der Daseinsvorsorge betrifft diese Dynamik auch die Siedlungswasserwirtschaft. Herausforderungen sind hier primär demographische Veränderungen, der Klimawandel, verschärfte gesetzliche Anforderungen, ein fortgeschrittener Investitionsstau und das wachsende Umweltbewusstsein von Bürgerinnen und Bürgern (Rost/Maier/Böhm et al. 2015; BMUB/ UBA 2017). Das konventionelle System der Abwasserbeseitigung erfährt damit einen zunehmenden Innovationsdruck. Einen konzeptionellen Lösungsansatz für die auftretenden systemischen Problemlagen stellen Neuartige Sanitärsysteme (NASS) dar (DWA 2010: 3; BMVI/BBSR 2016: 101). Bei Neuartigen Sanitärsystemen handelt es sich um einen Sammelbegriff für verschiedene Systemvarianten, bei denen ein ressourcenorientierter Umgang mit Abwasser im Mittelpunkt steht (DWA 2010: 7). Neben Antworten auf abwasserwirtschaftliche Fragestellungen können Neuartige Sanitärsysteme durch die vorgesehene Verwertung von Abwasserinhaltsstoffen zur Optimierung regionaler Ressourcenkreisläufe beitragen (DWA 2014: 8). So kann etwa der im Abwasser befindliche Phosphor zur anfallsortnahen Erzeugung von marktfähigen Düngesubstraten genutzt werden. Indem Ressourcen in der Region verbleiben und überregional zu vermarktende Produkte entstehen, können Neuartige Sanitärsysteme die regionale Wertschöpfung unterstützen.
Hinsichtlich der Verbreitung von Neuartigen Sanitärsystemen sind auf kommunaler Ebene kaum Schritte zur Realisierung zu beobachten (Kerber/Schramm/Winker 2016: 9). Die Ursachen sind vielfältig. So erfordert die Einführung von Neuartigen Sanitärsystemen einen grundlegenden Paradigmenwechsel in der Abwasserwirtschaft - weg von der Entsorgung des Abwassers, wie es bisher im herkömmlichen System erfolgt, hin zu dessen Verwertung. Damit ist ein tiefgreifender Systemwandel, eine Transformation der Siedlungswasserwirtschaft erforderlich. In seiner Gesamtheit besteht das Abwasserinfrastruktursystem jedoch aus technischen Komponenten unterschiedlicher Größe, Funktion, gesetzlicher Anforderung und Abschreibungszeit. Die Komplexität und Verknüpfung dieser Einheiten erschweren die Transformation. Zudem bedürfen Neuartige Sanitärsysteme der zunehmenden systemischen Vernetzung der Abwasser-, Abfall-, Land- und Energiewirtschaft sowie Modifikationen in den jeweiligen Rechtsbereichen. Die Realisierung ressourcenorientierter Abwassersysteme formuliert damit vielfältige Anforderungen an Akteure der Politik, Praxis und Zivilgesellschaft.

Der Ausbau des Abwasserinfrastruktursystems unterliegt keiner autonomen und unkontrollierten Entwicklung. Zwar kann dies aufgrund „kumulierter Trägheitsmomente und der ausgeprägten Pfadabhängigkeiten" (Hiessl/Toussaint/Becker et al. 2003: 134) so erscheinen, in der Realität handelt es sich aber um ein beeinflussbares "gesellschaftliches Konstrukt" (Hiessl/ Toussaint/Becker et al. 2003: 134), wobei die Steuerung des Ausbaus der Abwasserinfrastruktur eine zentrale Aufgabe der Länder darstellt. In diesem Kontext verfolgt der Beitrag das Ziel darzulegen, inwieweit durch den strategischen Einsatz des bestehenden wasserwirt- 
schaftlichen Instrumentariums die Einführung von Neuartigen Sanitärsystemen auf Landesebene unterstützt werden kann. Als Leitmotiv gilt, Perspektiven zur Realisierung integrierter Systemansätze in der Abwasserwirtschaft aufzuzeigen. So soll auch ein Beitrag zu der in der Raumplanung geführten Diskussion über Ansatzpunkte einer nachhaltigen Entwicklung strukturschwacher Regionen erbracht werden.

Als Modellgemeinde der Untersuchung dient die in Thüringen gelegene Gemeinde Rohrbach. Das Forschungsdesign setzt sich aus den zwei zentralen methodischen Bausteinen einer Methodentriangulation (vgl. Gläser/Laudel 2010; Flick 2011) und Konstellationsanalyse (vgl. Schön/Kruse/Meister et al. 2007) zusammen. Bei der Methodentriangulation erfolgte bezogen auf die siedlungswasserwirtschaftlichen Zusammenhänge eine umfassende Auswertung wissenschaftlicher Fachliteratur, Projektberichte anderer NASS-Vorhaben sowie der wasserrechtlichen Regularien. Die potenzielle Realisierbarkeit Neuartiger Sanitärsysteme für die Gemeinde Rohrbach wurde im Rahmen einer Konstellationsanalyse untersucht. Hier wurden mehr als 20 systematisierte Experteninterviews (vgl. Bogner/Littig/Menz 2002: 37) geführt und mit einer regelgeleiteten qualitativen Inhaltsanalyse (vgl. Gläser/Laudel 2010) ausgewertet. Dieses Vorgehen ermöglicht eine systematische Identifikation und Klassifizierung von bei der Realisierung von Neuartigen Sanitärsystemen auftretenden „Transformationsrisiken" (Kerber/Schramm/Winker 2016: 13). Im Rahmen einer weiterführenden Betrachtung wurden die Erkenntnisse auf Widersprüche und Redundanzen geprüft und durch Befunde aus der Fachliteratur verifiziert. Auf dieser Grundlage wurden Handlungsempfehlungen abgeleitet. Zu den Gesprächspartnern zählten Vertreterinnen und Vertreter verschiedener Verwaltungsressorts und Verwaltungsebenen, Wissenschaftlerinnen/Wissenschaftler, regionale Praxisakteure aus der Abwasser-, Abfall-, Land- und Energiewirtschaft sowie Bürgerinnen und Bürger.

Der Beitrag ist in sechs Kapitel gegliedert. Nach der Einführung werden in Kapitel 2 die grundlegenden Rahmenbedingungen der Siedlungswasserwirtschaft beschrieben. In Kapitel 3 werden das für die Modellgemeinde konzipierte Neuartige Sanitärsystem und die Transformationsrisiken erläutert, die dessen Einführung erschweren. In Kapitel 4 wird der Frage nachgegangen, über welche Instrumente die Thüringer Landeswasserbehörden verfügen und wie diese strategisch eingesetzt werden können, um den Transformationsrisiken zu begegnen. Auf dieser Grundlage werden in Kapitel 5 konkrete Handlungsempfehlungen abgeleitet und die Rolle der Raumplanung thematisiert. Abschließend erfolgt in Kapitel 6 ein Fazit.

\section{Rahmenbedingungen der Siedlungswasserwirtschaft}

In Deutschland stellt die Siedlungswasserwirtschaft mit der Wasserver- und Abwasserentsorgung einen Baustein der Daseinsvorsorge dar. Standen mit der Einführung des ableitungsorientierten Systemansatzes in der Mitte des 19. Jahrhunderts zunächst Entwässerungsund Hygienemaßnahmen im Vordergrund, umfassen die Ziele der heutigen integralen Siedlungswasserwirtschaft auch den Gewässerschutz und die Nutzungssicherung hinsichtlich der Trinkwasserversorgung (DWA 2006: 15-18). Zudem müssen entsprechende Dienstleistungen für die Bürgerinnen und Bürger dauerhaft zugänglich und bezahlbar sein (Libbe/Trapp/Winker 2017: 31).

Rechtlich wird auf der Grundlage der Europäischen Wasserrahmenrichtlinie (WRRL) ${ }^{1}$ ein guter Zustand aller Gewässer gefordert (vgl. WRRL Art. 4). In Deutschland soll dies insbesondere durch im Wasserhaushaltsgesetz $(\mathrm{WHG})^{2}$ verankerte Bestimmungen für die Einleitung von Abwasser umgesetzt werden. So müssen Abwasserbehandlungsanlagen dem Stand der Technik und andere Abwasseranlagen den allgemein anerkannten Regeln der Technik entsprechen ( $\S 60 \mathrm{WHG}$ ). Mit dem Stand der Technik und den allgemein anerkannten Regeln der Technik bedient sich der Gesetzgeber unbestimmter Rechtsbegriffe. Damit soll flexibel auf die sich ändernden Anforderungen in der Wasserwirtschaft reagiert und technische Neuerungen unkompliziert in die Praxis aufgenommen werden (Tauchmann/Hafkesbrink/Nisipeanu et al. 2006: 43).

Übernimmt der Bund mit dem Wasserhaushaltsgesetz eine übergeordnete regelnde Funktion, erfolgt die Steuerung des Ausbaus der Abwasserinfrastruktur auf der Landesebene. Zur Umsetzung der rechtlichen Vorgaben können die jeweiligen Landeswasserbehörden auf ein vielfältiges Instrumentarium zurückgreifen (vgl. Kapitel 4). Wie die meisten Bundesländer nutzt Thüringen eine dreistufige Verwaltungsorganisation. Somit liegt eine aufgabenorientierte Ausdifferenzierung an Fachauf-

1 Richtlinie 2000/60/EG des Europäischen Parlaments und des Rates vom 23. Oktober 2000 zur Schaffung eines Ordnungsrahmens für Maßnahmen der Gemeinschaft im Bereich der Wasserpolitik.

2 Wasserhaushaltsgesetz vom 31. Juli 2009, das zuletzt durch Artikel 2 des Gesetzes vom 4.Dezember 2018 geändert worden ist. 
sichtsbehörden vor. ${ }^{3}$ Landeseigene Facheinrichtungen wie das auch als obere Wasserwirtschaftsbehörde fungierende Thüringer Landesamt für Umwelt, Bergbau und Naturschutz (TLUBN) leisten zudem vollzugstechnische Unterstützung. ${ }^{4}$ Die Pflicht zur Beseitigung von Abwasser obliegt den Kommunen ( $\$ 56$ WHG in Verbindung mit $\S 47$ ThürWG $^{5}$ ). In der Praxis kann die Abwasserbeseitigung durch kommunale Eigenbetriebe oder im Rahmen einer interkommunalen Zusammenarbeit von Zweckverbänden übernommen werden ( 16 ThürKKG ${ }^{6}$ ).

Konzeptionell blieb das herkömmliche Abwasserinfrastruktursystem mit seinen wesentlichen Komponenten (Spültoilette, Schwemmkanalisation, Kläranlage) seit der Einführung unverändert (Tauchmann/Hafkesbrink/ Nisipeanu et al. 2006: 15). Bei bisher eingeführten Neuerungen handelte es sich ausschließlich um inkrementelle Innovationen, die problemlos in das konventionelle System integriert werden konnten (Hiessl/Toussaint/ Becker et al. 2003: 134). Die auftretenden sozioökonomischen, ökologischen und politischen Veränderungsprozesse verlangen nun nach einem tiefgreifenden Systemwandel (Hillenbrand/Niederste-Hollenberg/Menger-Krug et al. 2010; Libbe/Trapp/Winker 2017).

\section{Neuartige Sanitärsysteme als Systeminnovation}

In Expertenkreisen der Wasserwirtschaft und Raumplanung werden Neuartige Sanitärsysteme als ein geeigneter Lösungsansatz für die zukünftige Tragfähigkeit der Abwasserentsorgung im ländlichen Raum betrachtet (DWA 2010: 3; BMVI/BBSR 2016: 101). Der Leitgedanke von Neuartigen Sanitärsystemen liegt in einem systematisierten Ansatz zum stoff- und energieflussorientierten Umgang mit Abwasser aus zu Wohnzwecken genutzten Anlagen oder ähnlichen Herkunftsbereichen. Abwasserteilströme sollen separat erfasst, abgeleitet und behandelt werden. Differenziert wird im Wesentlichen zwischen dem aus der Toilette stammenden Schwarzwasser, Braunwasser (ausschließlich Fäzes mit Spülwasser), Gelbwasser (Urin mit Spülwasser) und dem in Bad und Küche anfallenden Grauwasser (DWA 2014:

$3 \mathrm{Vgl.} \mathrm{https://www.thueringen.de/th3/tmik/wir/index.aspx} \mathrm{(06.01.2020).}$ $4 \mathrm{Vgl}$. https://tlubn.thueringen.de/wasser/ (10.01.2020).

5 Thüringer Wassergesetz vom 28. Mai 2019.

6 Thüringer Gesetz über die kommunale Gemeinschaftsarbeit (ThürKGG) in der Fassung der Bekanntmachung vom 10. Oktober 2001, zuletzt geändert durch Artikel 5 des Gesetzes vom 23. Juli 2013.
8). Ziele von Neuartigen Sanitärsystemen sind insbesondere die adäquate Behandlung und Verwertung von Abwasserteilströmen, die Optimierung regionaler Stoffkreisläufe durch die Integration weiterer organischer Fraktionen (z. B. aus Abfall- und Landwirtschaft) sowie die intelligente Ergänzung und Umnutzung bestehender Infrastrukturen (DWA 2008: 22-24; DWA 2014: 8-9). Im Vergleich mit dem konventionellen System ist mit Neuartigen Sanitärsystemen die gleiche Entsorgungssicherheit gegeben (Kerber/Schramm/Winker 2016: 22), wobei Umweltbelangen besser entsprochen werden kann (DWA 2008). Zudem ist es möglich, mit neuen Wertschöpfungsketten und der Vermarktung entsprechender Produkte (etwa Düngesubstrate und Energie) einen Beitrag zur ökonomischen Stabilisierung strukturschwacher ländlicher Regionen zu leisten (Rost/Maier/Böhm et al. 2015: 349).

\subsection{Modellgemeinde Rohrbach}

Modellgemeinde für die Analyse von Hemmnissen bei der Einführung eines Neuartigen Sanitärsystems ist die etwa 20 Kilometer nördlich von Weimar gelegene Gemeinde Rohrbach. Trotz der unmittelbaren Nähe zur Thüringer Städtekette nahm die Bevölkerung Rohrbachs seit Mitte der 1990er-Jahre insgesamt um rund 20 Prozent ab, sodass aktuell knapp 200 Einwohner in der Gemeinde leben. ${ }^{7}$ Die zumeist in den 1960er-Jahren errichteten Anlagen der Abwasserentsorgung sind überwiegend sanierungsbedürftig und entsprechen in weiten Teilen nicht dem Stand der Technik. In Anbetracht dieser Rahmenbedingungen steht Rohrbach exemplarisch für viele Gemeinden des ländlichen Raums der neuen Bundesländer.

Den für Rohrbach entwickelten Konzeptansatz zeigt Abbildung 1. In diesem Ansatz ist für das gering belastete Grauwasser die Behandlung in einer kommunalen Pflanzenkläranlage mit Ableitung des gereinigten Abwassers in ein lokales Oberflächengewässer vorgesehen. Das nährstoffreiche und energetisch wertvolle Schwarzwasser soll mittels einer Unterdruckkanalisation zentral in Rohrbach gesammelt und in einer Biogasanlage in der Region verwertet werden. Aus Gründen der Ressourceneffizienz und der Wirtschaftlichkeit wird die Zugabe von weiteren organischen Fraktionen aus der Land- oder Abfallwirtschaft angestrebt. Somit können auf regionaler

7 Vgl. http://www.statistik.thueringen.de/datenbank/portrait.asp?ausw ahl=gem\&nr=71081\&vonbis=\&TabellelD=gg000102 (06.01.2020). 


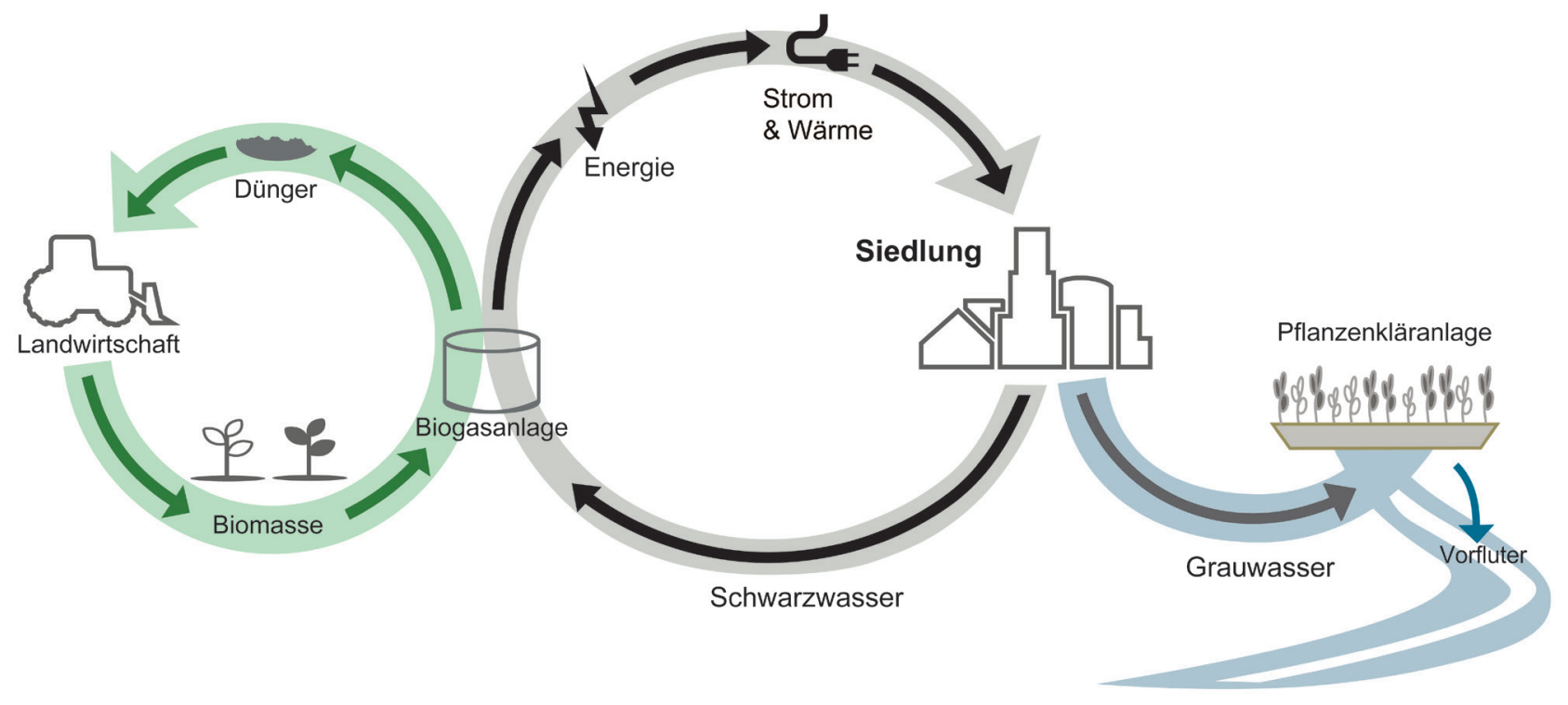

Abbildung 1: Konzeptansatz des Neuartigen Sanitärsystems für Rohrbach Quelle: Maier/Wolf/Londong (2017: 35), eigene Bearbeitung

Basis Energie (Wärme und Strom) sowie ein marktfähiges Düngeprodukt erzeugt werden.

Für die Realisierung des NASS-Ansatzes ist der Bau einer zentralen Pflanzenkläranlage, der Bau einer Unterdruckentwässerung, die Trennung der Abwasserteilströme, die Schwarzwasserbehandlung in einer Biogasanlage und die Sanierung alter Kanäle zur Ableitung des Niederschlagswassers vorgesehen (Hillenbrand/ Londong/Steinmetz et al. 2016: 995).

\subsection{Transformationsrisiken für Abwasserentsorger}

Bei der grundlegenden Realisierung der Abwasserinfrastruktur sind Neuartige Sanitärsysteme (noch) nicht die gängige Technik. Gleichwohl haben entsprechende Ansätze in verschiedenartigen Projekten ihre Praxistauglichkeit bewiesen (Libbe/Trapp/Winker 2017: 32). In Hinblick auf quartierübergreifende Ansätze sind beispielsweise Lübeck-Flintenbreite, DEUS 2 in Knittlingen und Hamburg-Jenfeld zu nennen (Hillenbrand/NiedersteHollenberg/Menger-Krug et al. 2010: 190). ${ }^{8}$ International sind in mehreren Städten Neuartige Sanitärsysteme in Planung (z. B. Paris und Stockholm), im Bau (z. B. Helsingborg) und in Betrieb (z. B. in den Niederlanden Sneek und Amsterdam-Buiksloterham).

8 Für Hamburg-Jenfeld vgl. http://www.jenfelderau-info.de/index. php/projekt.html (06.01.2020).
Die Realisierung von Neuartigen Sanitärsystemen ist für Aufgabenträger der Abwasserentsorgung mit vielfältigen Transformationsrisiken verbunden. Diese führen bei Wasserwirtschaftsakteuren zu einer abwartenden Haltung (Kerber/Schramm/Winker 2016: 13). Im Wesentlichen können „institutionelle Risiken“, „Regulationsrisiken“, "Legitimationsrisiken" und "ökonomische Risiken" auftreten (Kerber/Schramm/Winker 2016: 13 ff.). Des Weiteren zählen technische Risiken und Akzeptanzrisiken dazu (Hillenbrand/Niederste-Hollenberg/MengerKrug et al. 2010: 196; Albold/Büttner/Lamkowsky et al. 2016: 2; Schramm/Kerber 2017: 61). Diese sechs Risiken werden nachfolgend erläutert.

\subsubsection{Institutionelle Risiken}

Die Einführung von Neuartigen Sanitärsystemen erfordert einen integrativen und intersektoralen Planungsansatz der Infrastrukturentwicklung. So gilt es, etwa bei der Realisierung neuer Stadtquartiere, relevante Entscheidungsträger möglichst frühzeitig einzubeziehen. Vernetzt werden sollten etwa Vertreterinnen und Vertreter der Stadt- und Freiraumplanung, Architektur, Wasserver- und -entsorgung, Abfall- und Ressourcenwirtschaft sowie Landwirtschaft (DWA 2014: 29 f.). In der Praxis erschweren jedoch oftmals monodisziplinär verlaufende Planungsprozesse sektorübergreifende Infrastrukturlösungen (Libbe/Schramm/Winker et al. 2017: 83). So berichten Praxisakteure der Siedlungswasserwirtschaft, 
dass Vorhaben zumeist in einer konventionellen Planungsroutine von Stadt- und Regionalplanerinnen und -planern entworfen werden, wobei der Austausch mit Infrastrukturanbietern oder Genehmigungsbehörden erst im fortgeschrittenen Stadium des Planungsprozesses erfolgt. Darüber hinaus werden Wasserwirtschaftsakteure von kommunalen Akteuren ausschließlich als Dienstleister und nicht Impulsgeber für Konzeptansätze wahrgenommen. Die frühzeitige Integration von innovativen Ansätzen in Planungsprozesse ist damit nur bedingt möglich. Als weitere Hemmnisse werden das Fehlen eines koordinierenden und motivierenden Gremiums sowie konventionelle Ansprüche einzelner Akteure angesehen (Kerber/Schramm/Winker 2016: 14-15).

\subsubsection{Regulationsrisiken}

In Abhängigkeit des Abwasserteilstroms und anvisierten Verwendungszwecks kann der derzeitige rechtliche Rahmen ein wesentliches Hindernis darstellen. Die Ursache liegt insbesondere in fehlenden gesetzlichen Vorgaben hinsichtlich der Verwertung anfallender Abwasserströme (DWA 2010: 11). Entscheidungen für innovative Ansätze erweisen sich folglich als risikoreich (Schramm/Kerber 2017: 62). Zwar sind rechtliche Anforderungen etwa für die Grauwassernutzung (z. B. für Toilettenspülung und Bewässerung) oder die Vergärung und Energiegewinnung aus Schwarzwasser prinzipiell vorhanden (WSWU 2015: 134; Hanke 2016), für die stoffliche Verwertung von Schwarzwasser ist jedoch unklar, ob grundsätzlich das Abwasser- oder Abfallrecht anzuwenden ist (Schulz 2018: 214). Zudem zeigt sich, dass die bei Neuartigen Sanitärsystemen zur stofflichen Verwertung anfallenden Sekundärrohstoffe kaum in den entsprechenden Rechtsgrundlagen berücksichtigt werden (DWA 2014: 24). So wird beispielsweise Schwarzwasser nicht als Ausgangsstoff für Dünger aufgeführt (Schulz 2018: 214), gleichwohl es je nach Qualität einem Düngemittel nach $\S 2$ Abs. 1 Düngegesetz (DüngG) entsprechen könnte (WSWU 2015: 134). Dies trifft ebenso auf die Düngemittelverordnung (DüMV) zu. Folglich stehen den Absatz- und Verwertungswegen der NASS-Produkte keine rechtlichen Regelungen entgegen. Stattdessen wird die Ausbringung durch fehlende Begriffsbestimmungen eingeschränkt.

Mit der Einführung von Neuartigen Sanitärsystemen werden zudem die Systemgrenzen der Abwasserwirtschaft vom öffentlichen in den privaten Raum verschoben (Hanke 2017: 159). In der Regel unterliegt die Ausgestaltung der Abwasserinfrastruktur innerhalb von
Gebäuden und auf privaten Grundstücken den Eigentümern. Jedoch erfordern Neuartige Sanitärsysteme zur getrennten Erfassung und Ableitung der Abwasserteilströme Modifikationen an diesen Systemkomponenten. Zuständigkeitsfragen für die jeweilige Errichtung und Wartung der technischen Anlagen müssen folglich berücksichtigt werden (Schulz 2019: 47-48). Zudem sollte eine Überprüfung gebührentechnischer Belange erfolgen.

\subsubsection{Legitimationsrisiken}

Im Bereich der Abwasserwirtschaft wird die Realisierung von Innovationen überwiegend durch Entscheidungsträger der politischen Ebene und weniger durch den technischen Fortschritt bestimmt (Tauchmann/Hafkesbrink/ Nisipeanu et al. 2006). Hinsichtlich neuartiger Wasserinfrastrukturen fehlen jedoch politische Beschlüsse oder inhaltliche Auseinandersetzungen, welche deren Einführung unterstützen (Schramm/Kerber 2017: 63-64). Diese sind aber insbesondere im Hinblick auf den durch Neuartige Sanitärsysteme angestrebten stoff- und energieflussorientierten Umgang mit Abwasser erforderlich. Während die Aufgabe von Abwasserentsorgern bisher die Beseitigung von Abwasser ist ( $\$ 56$ WHG), wird innen im Rahmen von Neuartigen Sanitärsystemen zunehmend die Funktion eines ,Verwerters' zugeschrieben. Dies beinhaltet gesonderte Anforderungen an die Aufgabenorganisation bei der Abwasserentsorgung, sodass sich grundsätzlich die Frage nach geeigneten Betreibermodellen für Neuartige Sanitärsysteme stellt. So ist anzunehmen, dass für die Verwertung der Abwasserteilströme verstärkt private Partner aus der Landwirtschaft oder Energiewirtschaft mit einbezogen werden müssen. Im Mittelpunkt der zu führenden Diskussion müssen insbesondere Betreibermodelle für den Bau, den Betrieb und die Instandhaltung von intersektoral genutzten Infrastrukturanlagen sowie die Vermarktung der NASS-Produkte (z. B. hochwertiger Dünger) stehen. Einen Ansatz zur Modellfindung stellt das Drei-PhasenKooperationsmodell dar, das die wesentlichen Schritte für die Konzeption von NASS-Betreibermodellen aufzeigt (Schramm/Kerber/Trapp et al. 2018). Es bietet die Möglichkeit, die akteurspezifischen Formen der Zusammenarbeit für die Planungs-, Implementierungs- und Betriebsphase zu integrieren (Ebert/Schramm/Wang et al. 2019). 


\subsection{4 Ökonomische Risiken}

Im Vergleich zur konventionellen Abwasserinfrastruktur wird angenommen, dass die Realisierung eines Neuartigen Sanitärsystems mit höheren Investitionskosten verbunden ist (Schramm/Kerber 2017: 64). Grund hierfür sind weitreichende bauliche Veränderungen der Abwasserinfrastruktur inner- und außerhalb von Gebäuden (Hillenbrand/Niederste-Hollenberg/Menger-Krug et al. 2010: 193). Da erhöhte Finanzierungskosten von wasserwirtschaftlichen Akteuren als ein betriebswirtschaftliches Risiko wahrgenommen werden (Schramm/Kerber 2017: 64), wirken sich diese als ein eklatantes Hemmnis bei der Einführung neuer Infrastruktursysteme aus (Sartorius/Hillenbrand 2008: 386). Innovative Ansätze werden dementsprechend kaum forciert (Kerber/Lux 2016). Aktuelle Forschungsergebnisse zeigen jedoch, dass eine Stoffstromtrennung durch neue technische Komponenten zunehmend ohne signifikanten Umbaubedarf möglich ist (Berndt/Veser 2014; Schulz 2019: 48). Zudem deuten Studien an, dass Neuartige Sanitärsysteme mittel- und langfristig, betriebswirtschaftlich und insbesondere gesamtwirtschaftlich betrachtet auf vergleichbarem Niveau mit dem konventionellen System liegen (Bernhard/Felmeden/Kluge 2010). Bei entsprechenden umweltökonomischen Bilanzierungen ist neben der zeitlichen Ebene auch die räumliche ( $z$. B. Modell- oder Stadtebene) zu berücksichtigen (Zimmermann/Felmeden/Michel 2018).

\subsubsection{Technische Risiken}

In den vergangenen Jahren haben zahlreiche Demonstrationsvorhaben dazu beigetragen, die technischen Risiken von Neuartigen Sanitärsystemen zu reduzieren (Hillenbrand/Niederste-Hollenberg/Menger-Krug et al. 2010: 190). In der Praxis stehen für die technische Ausgestaltung verschiedene Systemkomponenten zur Erfassung und Ableitung von Abwasserteilströmen zur Verfügung. Dies trifft auch auf Behandlungsverfahren für die verschiedenen Stoffströme zu, von denen die meisten bereits im konventionellen System erprobt wurden (DWA 2014: 11-15). Gleichwohl entsprechen Neuartige Sanitärsysteme bisher noch nicht gänzlich dem Stand der Technik. (Albold/Büttner/Lamkovsky et al. 2016: 14). So sind insbesondere technische Normen erforderlich, für die es jedoch Erfahrungen aus dem praktischen Betrieb von Neuartigen Sanitärsystemen bedarf (Hillenbrand/ Niederste-Hollenberg/Menger-Krug et al. 2010: 196). Darüber hinaus besteht weiterhin Forschungsbedarf für einzelne Systemkomponenten wie beispielsweise die Stoffstromtrennung. ${ }^{9}$

\subsubsection{Akzeptanzrisiken}

Im Allgemeinen wird bei Wasserwirtschaftsakteuren von einer gesellschaftlichen Offenheit gegenüber neuartigen Wasserinfrastrukturen ausgegangen. Voraussetzung ist, dass die Technik verlässlich funktioniert und keine Komforteinbußen bei den Nutzerinnen und Nutzern auftreten (Kerber/Schramm/Winker 2016: 18). Studien hinsichtlich verschiedener Ansätze belegen, dass beispielsweise die hausinterne Grauwasserwiederverwendung ein hohes Akzeptanzpotenzial erfährt (Deffner/Birzle-Harder 2017: 147; Winker/Brüning/Meyer et al. 2019: 17) und sich die Nutzermehrheit trotz des Geräuschpegels prinzipiell wieder für eine Vakuumtoilette entscheiden würde (Hegger 2007: 127). In Rohrbach tritt ein Akzeptanzrisiko insbesondere bei der angestrebten landwirtschaftlichen Verwendung der erzeugten Düngesubstrate auf. So untersagen viele Landverpächter in der Modellregion aus Sorge vor Schadstoffen die Ausbringung von abwasserwirtschaftlichen Produkten. Darüber hinaus nehmen Großabnehmer aus Imagegründen und Akzeptanzbedenken auf Verbraucherebene entsprechend gedüngte landwirtschaftliche Produkte nicht an.

\section{Instrumente und Steuerungsansätze}

Landeswasserbehörden können auf ein umfassendes wasserwirtschaftliches Instrumentarium zurückgreifen, um die technisch-konzeptionelle Ausgestaltung der Abwasserentsorgung zu steuern (vgl. Tabelle 1). Die einzelnen Instrumente bieten hierbei die Chance für spezifische Steuerungsansätze, mit denen etwa die Thüringer Landeswasserbehörden auf die Transformationsrisiken reagieren können.

\subsection{Rechtliche Ausgestaltung}

In Ergänzung zum Wasserhaushaltsgesetz als Bundesgesetz wird das Wasserrecht in den Bundesländern durch

9 Vgl. z. B. https://www.uni-weimar.de/de/bauingenieurwesen/ professuren/siedlungswasserwirtschaft/forschung/aktuelleprojekte/awas/ (07.01.2020). 
Tabelle 1: Zentrale Instrumente der Landeswasserbehörden

\begin{tabular}{lll}
\hline Instrumente & Rechtliche Ausgestaltung & Thüringer Wasserrecht \\
& Satzungsrecht \\
Entscheidungsermessen & Entscheidungsspielräume \\
& Verwaltungsvorschriften \\
Ökonomische Regelungen & Abwasserabgabe \\
& Abwassersätze \\
& Fördermittel \\
& Strukturprogramme \\
& Wasser- und Bodenverbände \\
& Maßnahmenprogramme und Bewirtschaftungspläne \\
& Gewässerbeirat und Gewässerforen \\
\hline
\end{tabular}

die Landeswassergesetze geregelt. Die Aufgabe von Landeswassergesetzen liegt in der Ausgestaltung einer landesspezifischen Rechtsprechung entsprechend den wasserwirtschaftlichen Gegebenheiten. Hierfür können die Landesbehörden mit Ausnahme von anlagen- bzw. stoffbezogenen Regelungen vom Wasserhaushaltsgesetz abweichende landesrechtliche Vorschriften erlassen (Art. 72 GG). Zudem dürfen sie Gegenstände des Wasserrechts regeln, die vom Bundesgesetzgeber nicht berücksichtigt wurden. Folglich können die Thüringer Wasserbehörden mit dem Landeswassergesetz eine weitreichende rechtliche Konformität für Neuartige Sanitärsysteme schaffen. Eine Vorbildfunktion kann das im Jahr 2018 novellierte Hamburgische Abwassergesetz ${ }^{10}$ übernehmen. Indem der Begriff „Abwasserteilströme“ Eingang fand und der zuständige Abwasserentsorger in diesem Zusammenhang konkrete Anforderungen an die Einleitung von Abwasserteilströmen stellen kann (§ 11a Art. $5 \mathrm{HmbAbwG}$ ), wird die Einführung von Neuartigen Sanitärsystemen unterstützt. Zudem sollte mit dem Ziel der Verwertung von Abwasserteilströmen der Einsatz von Sonderklauseln geprüft werden. Anzudenken wäre etwa die Einführung eines ,Verwertungsparagrafen', der sich der normierten Tatbestandsvoraussetzung einer separaten Ableitung von Schwarzwasser anschließt. Vorerst formuliert mittels einer ,Soll-Vorschrift' (Erschließungsermessen), würde es den beteiligten Akteuren genügend Zeit einräumen, die benötigten Infrastrukturen zu realisieren.

10 Hamburgisches Abwassergesetz in der Fassung vom 14. Juli 2001, zuletzt geändert durch Artikel 5 des Gesetzes vom 23. Januar 2018.
Innerhalb der landesrechtlichen Grundlagen besteht auf örtlicher Ebene ein weiterer Regelungsbedarf (Tauchmann/Hafkesbrink/Nisipeanu et al. 2006: 43). Diesem wird mittels Bestimmungen in Gemeindeordnungen oder Satzungsrecht begegnet (Kopp-Assenmacher 2015: 462). Die wesentlichen Instrumente stellen Anschlussund Benutzungszwang dar, auf deren Grundlage Haushalte an ein Neuartiges Sanitärsystem angeschlossen werden können. Zudem sind Abwasserentsorger berechtigt, in Satzungen zu konstatieren, ,wie' das Abwasser durch die Haushalte zu übergeben ist (§ 58 Art. 2 ThürWG). Die separate Erfassung und Übergabe von Abwasserteilströmen kann somit eingefordert werden. Gemäß einer Duldungspflicht sind Abwasserentsorger zudem berechtigt, notwendige technische Systemkomponenten auf Privatgrundstücken zu installieren ( $\$ 20$ ThürKO) $)^{11}$, wobei entsprechende Begriffsbestimmungen in die Satzungen aufzunehmen sind (Schulz 2019: 46-47). Anfallende Mehrkosten würden durch Abwasserentsorger getragen und als Systemkosten mittels Abschreibungen in die Gebührenkalkulation einfließen.

\subsection{Entscheidungsermessen}

Das Handeln der Wasserbehörden wird durch bundesund landesgesetzliche Regelungen bestimmt. Zudem erfordern die differenzierten Entwicklungen von Regionen situationsbedingte Handlungsansätze, sodass der

11 Thüringer Gemeinde- und Landkreisordnung (Thüringer Kommunalordnung) in der Fassung der Bekanntmachung vom 28. Januar 2003, zuletzt geändert durch Artikel 2 des Gesetzes vom 16. Oktober 2019. 
Gesetzgeber Behörden zur Ausübung des Verwaltungsermessens verpflichtet (Schmidt 2007: 113). Damit verfügen Verwaltungen über ein Maß an Entscheidungsfreiheit, um sachgerecht und angemessen auf örtliche Gegebenheiten reagieren zu können (Starck 1991: 169). Als ein Instrument zum Lenken des Entscheidungsermessens können Ministerien oder Oberbehörden Verwaltungsvorschriften einsetzen (Peine 2008: 36). In Form von Erlassen, Richtlinien oder Anordnungen dienen sie nachgeordneten Instanzen als direkte Handlungsanweisungen (Frye 2012: 73-74). Aus sachlichen Gründen können sie dabei durch die erlassende Instanz jederzeit in ihren Bestimmungen geändert werden (Peine 2008: 36). Verwaltungsvorschriften bieten damit für die Länder die Möglichkeit, den konzeptionellen Ausbau der Abwasserinfrastruktur unmittelbar zu steuern.

Für Thüringen zeigt sich, dass bei den erlassenen Verwaltungsvorschriften der Abwasserwirtschaft zwischen Förderrichtlinien (vgl. Kapitel 4.3) und systemweisenden Verwaltungsvorschriften zu differenzieren ist. In Letzteren sind insbesondere Anforderungen an die Planung von Abwasserinfrastrukturprojekten festgelegt. Ein Beispiel für Thüringen sind die Anforderungen zur Aufstellung von Abwasserbeseitigungskonzepten (ABK) durch die Abwasserentsorger (TMLFUN 2012). Zwar schließt die entsprechende Verwaltungsvorschrift die Realisierung von Neuartigen Sanitärsystemen nicht unmittelbar aus, der konzeptionelle Fokus liegt jedoch auf der Verstetigung des konventionellen Systems. Grundsätzlich sollten die Anforderungen laut Kerber, Schramm und Winker (2016: 24) aber dauerhaft auf eine ökologisch nachhaltige Abwasserentsorgung ausgerichtet sein und vorausschauend klimatische und demographische Entwicklungsprozesse sowie den steigenden Ressourcen- und Energieverbrauch berücksichtigen. Diese Vorgaben wären in die entsprechenden Verwaltungsvorschriften aufzunehmen, sodass die Einführung einer ressourcenorientierten Abwasserbewirtschaftung eingefordert werden kann.

\section{3 Ökonomische Regelungen}

Im Zusammenspiel von Wasserhaushaltsgesetz und Abwasserabgabengeset $z^{12}$ wird dem Ziel der Verbesserung der Wassergüte und der Weiterentwicklung des

12 Gesetz über Abgaben für das Einleiten von Abwasser in Gewässer (Abwasserabgabengesetz - AbwAG) in der Fassung der Bekanntmachung vom 18. Januar 2005, das zuletzt durch Artikel 2 der Verordnung vom 22. August 2018 geändert worden ist.
Stands der Technik durch die Erhebung der Abwasserabgabe entsprochen. Die Höhe richtet sich nach der Schädlichkeit des eingeleiteten Abwassers und wird durch das Land erhoben (Nisipeanu 1991: 28; Köhler/ Meyer 2006: 20). Auf kommunaler Ebene umfasst das Satzungsrecht Gebührenregelungen, um eine Refinanzierung der jeweiligen abwasserwirtschaftlichen Aufwendungen zu gewährleisten (Tauchmann/Hafkesbrink/ Nisipeanu et al. 2006: 43). Mit dem Ziel, den Gedanken der Wertschöpfung in der Abwasserwirtschaft zu verankern, sollte das bestehende Abgabensystem um die Aspekte der Kreislaufwirtschaft und Wertschöpfung erweitert werden. Eine Möglichkeit bietet die Einführung einer gestaffelten Abwasserabgabe, die etwa finanzielle Anreize bei der Verwertung von Wasser und seiner Inhaltsstoffe gewährt. Zudem ist es für Abwasserentsorger möglich, Abwassergebühren innerhalb des eigenen Wirkungskreises anhand verschiedener Maßstäbe zu kalkulieren. Als ein solcher Maßstab kann die Beanspruchung von Behandlungsanlagen gewertet werden. Indem bei Haushalten mit Stoffstromtrennung eine effizientere Behandlung bzw. Verwertung des Abwassers möglich ist, könnten die Abwassergebühren gesondert berechnet werden.

Die Erweiterung des Abgabensystems sollte mit der Neuorientierung der Förderpolitik einhergehen. So weisen Praxisakteure der Siedlungswasserwirtschaft auf Fördermittel als wichtigen Impuls für die Durchführung innovativer Projekte hin (Schramm/Kerber 2017: 59). In Thüringen ist die Verwendung des Aufkommens aus der Abwasserabgabe zur Erweiterung von zentralen und dezentralen Kläranlagen sowie die Förderung von Pilotanlagen möglich (TMUEN 2018a; TMUEN 2018b; TMUEN 2018c). Die Förderpolitik sollte jedoch nicht nur um innovative Handlungsschwerpunkte ergänzt werden, welche einer ressourcenorientierten Abwasserentsorgung dienlich sind. Stattdessen sollte die Förderung konventioneller Lösungen reduziert werden. Finanzielle Ressourcen könnten somit gebündelt und innovative Ansätze präferierende Abwasserentsorger umfassend bei der Risikobewältigung unterstützt werden.

\subsection{Strukturierende Mechanismen}

In Anbetracht der Gebote der Kostenminimierung, der effektiveren Aufgabenwahrnehmung und der optimierten Ressourcennutzung gewinnen umfassende Organisationseinheiten in der Wasserwirtschaft zunehmend an Bedeutung (Tauchmann/Hafkesbrink/Nisipeanu et al. 2006). Orientieren sich die Thüringer wasserwirtschaft- 
lichen Organisationseinheiten bisher überwiegend an Kommunalgrenzen (TLUG 2009), sollte eine Umstrukturierung anhand von räumlichen und funktionalen Einheiten oder der in der Wasserrahmenrichtlinie ausgewiesenen Flusseinzugsgebiete erfolgen. Erreicht werden kann dies durch Landesprogramme, welche freiwillige Zusammenschlüsse von Abwasserentsorgern fördern (TLUG 2009: 4). Optional kann auch die Kommunalaufsicht auf der Grundlage des Gesetzes über die kommunale Gemeinschaftsarbeit ${ }^{13}$ und der Thüringer Kommunalordnung Fusionen veranlassen. Voraussetzung für eine strategische Umstrukturierung sind übergeordnete Leitbilder und an Zusammenschlüsse formulierte Anforderungen (vgl. Graetz 2008). Im Rahmen der durch Neuartige Sanitärsysteme vorgesehenen regionalen Wertschöpfung sollte zusätzlich eine Erweiterung der Organisationseinheiten um Regionalakteure der Land-, Abfall- und Energiewirtschaft erfolgen. Das Einbinden von bestehenden lokalen Zusammenschlüssen kann dabei den Vernetzungsprozess fördern (Rost/Maier/Böhm et al. 2015). Das in Thüringen mögliche Bilden von Wasserund Bodenverbänden ( $§ 47$ ThürWG) sollte hierbei als ein erster Schritt angesehen werden. Laut Tauchmann, Hafkesbrink, Nisipeanu et al. (2006: 69) bieten aber insbesondere sondergesetzliche Regelungen wie in Nordrhein-Westfalen die Möglichkeit, Abwasserentsorger und private Unternehmen in Genossenschaftsform zu organisieren und damit eine nachhaltigere Bewirtschaftung zu erreichen. Gesellschaftliche Hemmnisse, die bei entsprechenden Umstrukturierungsprozessen potenziell auftreten können, sollten als temporäre Ereignisse betrachtet werden.

\subsection{Planung und Beteiligung}

Auf Landesebene wird die Gewässerbewirtschaftung durch Programme und Wasserrahmenpläne konkretisiert (§§ 82, 83 WHG). Thüringen verfügt hierbei über das Landesprogramm "Gewässerschutz 2016-2021“ (TMUEN 2016). In Zusammenhang mit den Bewirtschaftungsplänen für die jeweiligen Flussgebietseinheiten (TMUEN 2016: 12) hat das Strategiepapier zwar keine rechtlich bindende Funktion, dient aber der wasserwirtschaftlichen Maßnahmenplanung als inhaltliche Orientierung (TMUEN 2016: 8). Die Beteiligung der

13 Thüringer Gesetz über die kommunale Gemeinschaftsarbeit (ThürKGG) in der Fassung der Bekanntmachung vom 10. Oktober 2001, zuletzt geändert durch Artikel 5 des Gesetzes vom 23. Juli 2013.
Öffentlichkeit wird dabei grundsätzlich als eine essenzielle Komponente für das Zustandekommen von Maßnahmen in der Wasserwirtschaft betrachtet. Das Land Thüringen verfügt zudem über den regelmäßig tagenden Gewässerbeirat und vereinzelte regionale Gewässerforen, in denen unter anderen Repräsentanten der Landwirtschaft, Abwasserwirtschaft, Kommunalverwaltung, Raumplanung und Bürgerschaft vertreten sind (TMUEN 2016: 22).

Die vorliegenden Planungs- und Beteiligungsstrukturen gilt es, im Sinne der Verbreitung von Neuartigen Sanitärsystemen zu nutzen und zu erweitern. So sollte das Landesprogramm Gewässerschutz der integrierten Strategiefindung dienen. Neuartige Sanitärsysteme können als ein zentraler Lösungsansatz für die abwasserwirtschaftlichen Herausforderungen in ländlichen Regionen aufgezeigt werden. Mittels des Gewässerbeirats sowie regionalen Gewässerforen können kommunale Vertreter, Repräsentanten der Abwasser-, Land-, Abfall- und Energiewirtschaft sowie Bürgerinnen und Bürger im Rahmen der Realisierung jeweils ortspezifischer Neuartiger Sanitärsysteme vernetzt werden.

\section{Handlungsempfehlungen}

\subsection{Möglichkeitsräume der Siedlungswasserwirtschaft}

Auf der Grundlage der dargestellten Instrumente wird deutlich, dass Landeswasserbehörden über ein vielfältiges Repertoire verfügen, um mittels strategischer Steuerungsansätze die Einführung einer ressourcenorientierten Abwasserwirtschaft zu unterstützen.

In Hinblick auf die zeitnahe Einführung des Neuartigen Sanitärsystems für die Gemeinde Rohrbach bedarf es des gesonderten, projektbezogenen Einsatzes ausgewählter Instrumente. Aufgrund der langjährigen Novellierungsprozesse von landes- und bundesgesetzlichen Regelungen im Bereich der Wasserwirtschaft sollte die Realisierung als Pilot- und Demonstrationsvorhaben erfolgen. Zwar finden sich im Thüringer Wassergesetz keine Hinweise auf den rechtlichen Umgang mit Pilotprojekten, im Rahmen des anzuwendenden Ermessensspielraumes können diese Vorhaben aber einen rechtlichen Sonderstatus erhalten. Dieser ermöglicht es auch, rechtliche Hemmnisse zur Verwertung von Schwarzwasser zu lösen, Kooperationen zwischen regionalen Akteuren im Sinne der integrierten Ressourcenwirtschaft zu bilden und Erkenntnisse für den Betrieb eines Neuarti- 
Tabelle 2: Handlungsempfehlungen für Akteure der Thüringer Landesverwaltung zur Einführung von Neuartigen Sanitärsystemen

Konzentration auf den Umbau der Abwasserinfrastruktur

- Integrierte Strategieformulierung mit Fokus auf ressourcenorientierten Systemansätzen

- $\quad$ Anpassung von systemweisenden Verwaltungsvorschriften (z. B. Berücksichtigung von Neuartigen Sanitärsystemen bei Hinweisen zur Erstellung von Abwasserbeseitigungskonzepten)

- $\quad$ Erarbeitung eines Kriterienkatalogs zur Ermittlung von NASS-geeigneten Regionen und Kommunen

- $\quad$ Einsatz eines multikriteriellen Bewertungsinstruments bei der Bewertung von Maßnahmen

- $\quad$ Sensibilisierung von Entscheidungsträgern und Bürgerinnen/Bürgern durch Bildungs- und Informationsarbeit

- Erforschung und Entwicklung von kostengünstigen und nutzerfreundlichen Systemkomponenten und Behandlungsverfahren

Anpassung von ober- und untergesetzlichen Regelwerken

- $\quad$ Verankerung des Kreislaufwirtschaftsgedankens in den abwasserwirtschaftlichen Gesetzesgrundlagen (Landes- und Kommunalrecht)

- $\quad$ Definition von Abwasserteilströmen und NASS-Komponenten sowie Einführung eines Verwertungsparagrafen für erfasste Abwasserteilströme im Thüringer Landeswassergesetz

- $\quad$ Erstellung von rechtlich geprüften Beispielformulierungen für Satzungsänderungen (Entwässerungs- und Gebührensatzung) als rechtliche Hilfestellung für Abwasserentsorger

- Schaffung von sondergesetzlichen Regelungen als Voraussetzung für Kooperationen verschiedener Akteure (u.a. öffentliche Aufgabenträger und privatwirtschaftliche Unternehmen) im Rahmen der regionalen Ressourcenverwertung

- $\quad$ Einführung von Qualitätszertifikaten für NASS-Düngesubstrate und Erweiterung normgebender Regelwerke

Neuausrichtung des Abgabensystems und der Förderpolitik

- $\quad$ Einführung eines Abgabensystems, das Wertschöpfungsaspekte berücksichtigt und damit für Abwasserentsorger und Bürgerinnen/Bürger finanzielle Anreize zur Einführung von Neuartigen Sanitärsystemen gibt

- Förderschwerpunktsetzung auf innovative Systemansätze

- $\quad$ Verminderte Förderung konventioneller Abwasserinfrastrukturvorhaben

- Ressortübergreifende Bündelung finanzieller Ressourcen (insbesondere Landwirtschafts- und Wirtschaftsförderung)

Bildung von leistungsstarken Organisationseinheiten

- $\quad$ Etablierung eines Leitbildes und Maßnahmenplanes zur Zusammenführung von Abwasserentsorgern anhand von Flusseinzugsgebieten sowie räumlich-funktionalen Gegebenheiten

- $\quad$ Bildung fachübergreifender Organisationseinheiten mit Regionalakteuren aus der Abwasser-, Land-, Abfall- und Energiewirtschaft

gen Sanitärsystems zu sammeln. Auf untergesetzlicher Ebene ist der zuständige Abwasserentsorger bei den notwendigen Satzungsänderungen durch die Landesbehörden zu unterstützen. In Abstimmung mit den ansässigen Wasserbehörden kann ein Neuartiges Sanitärsystem zudem als abwasserwirtschaftliche Maßnahme im Abwasserbeseitigungskonzept und damit auch im Investitionsplan des Abwasserentsorgers verankert werden. Neben Eigenmitteln sollten insbesondere Fördermittel die Finanzierung absichern. Für die systematische Realisierung von Neuartigen Sanitärsystemen sind darüber hinaus weitreichendere Schritte erforderlich (vgl. Tabelle 2).

Mit dem strategischen Einsatz der dargestellten Instrumente können die Landeswasserbehörden die systematische Einführung von Neuartigen Sanitärsystemen weitreichend unterstützen. Zur Überwindung einzelner Transformationsrisiken sind sie aber auf das Zusammenwirken mit anderen Fachressorts angewiesen. Primär ist eine intensive Zusammenarbeit mit dem Innenministerium als Aufsichtsbehörde der Gemeinden erforderlich.
So unterliegt die technisch-konzeptionelle Planung der Abwasserinfrastruktur zwar den Abwasserentsorgern und Wasserbehörden, die haushaltswirtschaftliche Prüfung entsprechender Maßnahmen erfolgt aber durch die Kommunalaufsicht. ${ }^{14}$ Die Kommunalaufsicht in Thüringen orientiert sich an den Haushaltsgrundsätzen der Sparsamkeit und Wirtschaftlichkeit ( $§ 53$ Abs. 2 ThürKO). Da Neuartige Sanitärsysteme im Vergleich zu konventionellen Maßnahmen jedoch mit einem erhöhten Investitionsvolumen einhergehen können, ist an dieser Stelle der erweiterte Nutzen ressourcenorientierter Systeme zu berücksichtigen. Durch das Innenministerium erlassene Verwaltungsvorschriften können die Kommunalaufsicht hierbei zu einer entsprechenden Handlungspraxis veranlassen. Auch in Zusammenhang mit dem zu modifizierenden Satzungsrecht und der Fusion von Abwasserentsorgern zu größeren Organisationseinheiten ist ein strategisches Zusammenwirken erforderlich.

14 Vgl. https://www.thueringen.de/th3/tlvwa/inneres_kommunales/ kommunalaufsicht/index.aspx (07.01.2020). 
Darüber hinaus erweist sich hinsichtlich der Ausbringung der NASS-Substrate die Kooperation mit den Landwirtschaftsbehörden als Vorteil. Erzeugte Düngesubstrate können vorerst auf landeseigenen Testflächen ausgebracht werden. Damit kann die Düngewirkung der Substrate in Langzeitversuchen erforscht werden, um diese in einem anschließenden Schritt als offizielles Düngemittel registrieren zu lassen.

Im Hinblick auf die durch Großabnehmer erwarteten Akzeptanzprobleme bei Verbraucherinnen und Verbrauchern hinsichtlich der landwirtschaftlichen Verwertung der Düngesubstrate deutet eine Untersuchung eine größere Offenheit an. So gab eine Auswahl an befragten Landwirten an, sich den Einsatz eines urinbasierten Düngers sowie den Verzehr der gedüngten Produkte grundsätzlich vorstellen zu können (Spoth/Schmidt/ Arnold 2012: 110). Gleichwohl sollten für die Herstellung einer grundlegenden gesellschaftlichen Akzeptanz weitere Kooperationen entstehen. Eine zentrale Rolle spielt dabei die durch technisch-wissenschaftliche Fachverbände organisierte Vergabe von Zertifikaten und freiwilligen Gütesiegeln. So wird etwa die Qualität von Klärschlämmen durch das von der gleichnamigen Gesellschaft vergebene Zertifikat "Qualitätssicherung Landbauliche Abfallverwertung" (QLA) gesichert (QLA 2017). Ein ähnliches Zertifikat wäre auch für NASSProdukte denkbar. Darüber hinaus sollten ,Designerdünger' ohne offensichtlichen Zusammenhang zu Fäkalien hergestellt werden. Gute Beispiele hierfür sind der klärschlammbasierte Dünger „Berliner Pflanze“15 und der aus Urin erzeugte Flüssigdünger „Aurin“. ${ }^{16}$ Kann mit Gütesicherungssystemen wie dem QLA-Zertifikat bei Landverpächtern und Großabnehmern die Akzeptanz erhöht werden, richten sich freiwillige Gütesiegel in der Lebensmittelbranche an die Bürgerinnen und Bürger. Für Thüringen ist das vom Landwirtschaftsministerium etablierte Siegel „Geprüfte Qualität aus Thüringen“ zu nennen. ${ }^{17}$ Eine Erweiterung um den Einsatz von NASSProdukten wäre zu prüfen. In Bezug auf die rechtlichen Fragestellungen werden darüber hinaus weitere Einschränkungen deutlich. Dies betrifft zum einen die im Wasserhaushaltsgesetz festgehaltenen anlagen- bzw. stoffbezogenen Regelungen (Kopp-Assenmacher 2015: 461). In Verbindung mit den bundesgesetzlichen düngemittelrechtlichen Vorschriften schränken diese die

15 http://www.bwb.de/de/6946.php (07.01.2020).

$16 \mathrm{https} / / / w w w . e a w a g . c h / d e / a b t e i l u n g / e n g / p r o j e k t e / a u r i n-$ duenger-aus-urin/ (07.01.2020).

17 https://www.agrarmarketing-thueringen.de/agrarmarketing/ qualitaetszeichen-nutzer.html (07.01.2020).
Verwertung der Abwasserteilströme und folglich die Einführung von Neuartigen Sanitärsystemen bisher ein. Die politische Auseinandersetzung auf der Landesebene sowie erste konzeptionelle Vorstöße zur Einführung von Neuartigen Sanitärsystemen können hierbei wichtige Impulse für notwendige Novellierungsverfahren auf der Bundesebene sein.

\subsection{Chance für die Raumplanung}

Mit strategischen Steuerungsansätzen können die Thüringer Landeswasserbehörden Ansätze Neuartiger Sanitärsysteme zwar als Pilot- und Demonstrationsvorhaben realisieren, die systematische Einführung erfordert aber das Zusammenwirken mit anderen Fachressorts und Gesellschaftsbereichen. So sollten nicht nur die Fachbehörden der Abwasser-, Abfall-, Land- und Energiewirtschaft kooperieren, sondern auch privatwirtschaftliche und zivilgesellschaftliche Institutionen dieser Sektoren wie Landwirtschaftsbetriebe oder Bürgerenergiegenossenschaften in Umsetzungsverfahren einbezogen werden. Zur Koordination dieses Prozesses bedarf es eines Akteurs mit ausgewiesenen organisatorischen und kommunikativen Fähigkeiten. In Anbetracht dieser Anforderungen und der gesellschaftspolitischen Position kann die Raumplanung hier einen wesentlichen Beitrag leisten (vgl. ARL 2011). Mit dem Ziel der Verknüpfung verschiedener Bereiche der Daseinsvorsorge können Planerinnen und Planer die spezifischen Verwaltungsressorts für die Anforderungen von Neuartigen Sanitärsystemen sensibilisieren und die ressortübergreifende Verwaltungsoptimierung unterstützen. In Verbindung mit dem integrierenden Verwaltungshandeln bedarf es der intersektoralen Vernetzung auch auf der Praxisebene. Zusätzlich sind Kooperationen von Wasserwirtschaftsakteuren mit privatwirtschaftlichen Entscheidern und Bürgerinnen/Bürgern aufzubauen. Die Raumplanung kann hier bei der Erarbeitung von regionalen Leitbildern und Handlungsstrategien oder integrierten Stadt- und Ortsentwicklungskonzepten Neuartige Sanitärsysteme frühzeitig in den Fokus von kommunalen Akteuren rücken und bei den zu führenden Aushandlungsprozessen eine moderierende Funktion einnehmen.

Im Rahmen der im Grundgesetz festgelegten kommunalen Selbstverwaltungsgarantie, auf deren Grundlage Gemeinden ihre städtebauliche Entwicklung selbstständig lenken, können Planerinnen und Planer die Einführung von Neuartigen Sanitärsystemen durch entsprechende Regelungen auf der Basis des Baugesetzbuches im besonderen Städtebaurecht unterstüt- 
zen. Auch Städtebauliche Verträge bieten die Chance, mittels konkreter Vereinbarungen zwischen privaten und kommunalen Akteuren die Einführung von Neuartigen Sanitärsystemen zu fördern.

Im Zusammenspiel mit der Siedlungswasserwirtschaft bietet sich für die Raumplanung ein weiterer Handlungsansatz an, um der in vielen ländlichen Teilräumen zunehmenden „De-Infrastrukturalisierung“ (Kersten/ Neu/Vogel 2012: 68) zu begegnen. Der vielerorts zu führende gesellschaftliche Aushandlungsprozess hinsichtlich der Neudefinition von Aufgaben und Zuständigkeiten sowie der Einbindung fachübergreifender Netzwerke als Voraussetzung für die Sicherung der Daseinsvorsorge (Beauftragter der Bundesregierung für die Neuen Bundesländer 2011: VI) kann somit einen wichtigen Impuls erfahren.

\section{Fazit}

Neuartige Sanitärsysteme (NASS) stellen einen geeigneten Lösungsansatz dar, die Tragfähigkeit der Abwasserentsorgung in ländlichen Räumen zu bewahren. Zudem können sie zur regionalen Wertschöpfung beitragen. Die Einführung ressourcenorientierter Systeme wird jedoch durch vielfältige Transformationsrisiken erschwert. Um diesen entgegenzuwirken und damit Aufgabenträger der Abwasserentsorgung bei der Realisierung von Neuartigen Sanitärsystemen zu unterstützen, können die Thüringer Wasserbehörden auf ein umfassendes Instrumentarium zurückgreifen. Indem der konzeptionelle Fokus in Thüringen auf der Verstetigung des bestehenden konventionellen Systems liegt, kann die Transformation hin zu einer ressourcenorientierten Siedlungswasserwirtschaft jedoch nicht durch vereinzelte Steuerungsansätze erreicht werden. Stattdessen bedingt die Überwindung der Transformationsrisiken den gebündelten und aufeinander abgestimmten Einsatz der verschiedenen wasserwirtschaftlichen Instrumente. Da Neuartige Sanitärsysteme zudem intersektorale Verknüpfungen erfordern und damit die abwasserwirtschaftlichen Systemgrenzen überschritten werden, bedarf es zur systematischen Einführung das Zusammenwirken verschiedener Verwaltungs- und Praxisakteure. Bei den zu führenden Aushandlungsprozessen kann die Raumplanung die Siedlungswasserwirtschaft entscheidend unterstützen.

Danksagung: Ein besonderer Dank gilt dem Bundesministerium für Bildung und Forschung (BMBF) für die finanzielle Förderung des Projektes "Neuorganisation
Ländlicher Abwasserentsorgung" (NoLA). Die Veröffentlichung des Beitrags wurde durch das Programm „Open Access Publizieren“ der Deutschen Forschungsgemeinschaft (DFG) und den Publikationsfonds der BauhausUniversität Weimar gefördert.

\section{Literatur}

Albold, A.; Büttner, S.; Lamkowsky, G.; Lange, A.; Stich, G. (2016): Abschlussbericht zum DBU-Projekt Qualifizierung und Vernetzung von Fachleuten im Bereich zukunftsfähiger Siedlungswasserwirtschaft. Leipzig.

ARL - Akademie für Raumforschung und Landesplanung (2011): Strategische Regionalplanung. Hannover. = Positionspapier aus der ARL 84.

ARL - Akademie für Raumforschung und Landesplanung (2016): Daseinsvorsorge und gleichwertige Lebensverhältnisse neu denken. Hannover. = Positionspapier aus der ARL 108.

Beauftragter der Bundesregierung für die Neuen Bundesländer (2011): Daseinsvorsorge im demografischen Wandel zukunftsfähig gestalten. Handlungskonzept zur Sicherung der privaten und öffentlichen Infrastruktur in vom demografischen Wandel besonders betroffenen ländlichen Räumen. Berlin.

Berndt, M.; Veser, S. (2014): EVaSENS - Neue Wege der Abwassertrennung im Siedlungsbestand. In: Wasser und Abfall 16, 3, 32-36.

Bernhard, M.; Felmeden, J.; Kluge, T. (2010): Bilanzierung und Bewertung bestehender und neuartiger Wasserinfrastrukturen. In: Kluge, T.; Libbe, J. (Hrsg.): Transformationsmanagement für eine nachhaltige Wasserwirtschaft. Handreichung zur Realisierung neuartiger Infrastrukturlösungen im Bereich Wasser und Abwasser. Berlin, 39-78.

BMUB - Bundesministerium für Umwelt, Naturschutz, Bau und Reaktorsicherheit; UBA - Umweltbundesamt (Hrsg.) (2017): Umweltbewusstsein in Deutschland 2016. Ergebnisse einer repräsentativen Bevölkerungsumfrage. Berlin.

BMVI - Bundesministerium für Verkehr und digitale Infrastruktur; BBSR - Bundesinstitut für Bau-, Stadt- und Raumforschung (Hrsg.) (2016): Regionalstrategie Daseinsvorsorge. Leitfaden für die Praxis. Berlin.

Bogner, A.; Littig, B.; Menz, W. (2002): Das Experteninterview. Theorie, Methode, Anwendung. Wiesbaden. doi: 10.1007/9783-322-93270-9

Deffner, J.; Birzle-Harder, B. (2017): Betriebswasserversorgung und Wärmerückgewinnung aus Einwohnersicht. In: Winker, M.; Trapp, J. H.; Libbe, J.; Schramm, E. (Hrsg.): Wasserinfrastruktur: Den Wandel gestalten. Technische Varianten, räumliche Potenziale, institutionelle Spielräume. Berlin, 143-158. = Edition Difu 16.

DWA - Deutsche Vereinigung für Wasserwirtschaft, Abwasser und Abfall (2006): Arbeitsblatt DWA-A 100. Leitlinien der integralen Siedlungsentwässerung. Hennef.

DWA - Deutsche Vereinigung für Wasserwirtschaft, Abwasser und Abfall (2008): Neuartige Sanitärsysteme. Hennef. = DWAThemen 12/2008. 
DWA - Deutsche Vereinigung für Wasserwirtschaft, Abwasser und Abfall (2010): Im Klartext. Brauchen wir in Deutschland neuartige Sanitärsysteme? Hennef.

DWA - Deutsche Vereinigung für Wasserwirtschaft, Abwasser und Abfall (2014): Arbeitsblatt DWA-A 272. Grundsätze für die Planung und Implementierung Neuartiger Sanitärsysteme (NASS). Hennef.

Ebert, B.; Schramm, E.; Wang, B.; Winker, M. (2019): Governance instruments for optimising source separation in novel urban water systems: the case of cross-connections in urban water systems. In Water Policy 21, 2, 412-427. doi: 10.2166/ wp.2019.183

Flick, U. (2011): Triangulation. Eine Einführung. Wiesbaden. = Qualitative Sozialforschung 12.

Frye, B. (2012): Verwaltungsvorschriften: Begriff, Funktion, Arten und Wirkungen. In: Thüringer Verwaltungsblätter 21, 4, 73-78.

Gläser, J.; Laudel, G. (2010): Experteninterviews und qualitative Inhaltsanalyse als Instrumente rekonstruierender Untersuchungen. Wiesbaden.

Graetz, H. (2008): Synergiepotenzial einer fragmentierten Wasserwirtschaft. Ein Beitrag zum Wert des Zusammenwirkens in fragmentierten Organisationsstrukturen der Wasserwirtschaft. Dissertation an der Bauhaus-Universität Weimar.

Hanke, S. (2016): Rechtliche Rahmenbedingungen neuartiger Wasserinfrastrukturen. Zu den rechtlichen Möglichkeiten und Grenzen der Einführung von Grauwasserrecycling, Schwarzwasserbehandlung sowie Wärmerückgewinnung. Berlin. = netWORKS-Paper 31 .

Hanke, S. (2017): Rechtliche Rahmenbedingungen neuartiger Wasserinfrastrukturen. In: Winker, M.; Trapp, J. H.; Libbe, J.; Schramm, E. (Hrsg.): Wasserinfrastruktur: Den Wandel gestalten. Technische Varianten, räumliche Potenziale, institutionelle Spielräume. Berlin, 159-170. = Edition Difu 16.

Hegger, D. (2007): Greening sanitary systems: an end-user perspective. Dissertation an der Universiteit Wageningen.

Hiessl, H.; Toussaint, D.; Becker, M.; Dyrbusch, A.; Geisler, S.; Herbst, H.; Prager, J. U. (2003): Alternativen der kommunalen Wasserversorgung und Abwasserentsorgung. AKWA 2100. Heidelberg. = Schriftenreihe des Fraunhofer-Instituts für Systemtechnik und Innovationsforschung 53. doi: 10.1007/9783-7908-2717-0

Hillenbrand, T.; Londong, J.; Steinmetz, H.; Wilhelm, C.; Sorge, C.; Söbke, H.; Nyga, I.; Minke, R.; Menger-Krug, E. (2016): Anpassung an neue Herausforderungen - nachhaltige Wasserinfrastruktursysteme für Bestandsgebiete. Ergebnisse des Forschungsvorhabens TWIST++. In: Korrespondenz Abwasser Abfall 63, 11, 992-998.

Hillenbrand, T.; Niederste-Hollenberg, J.; Menger-Krug, E.; Klug, S.; Holländer, R.; Lautenschläger, S.; Geyler, S. (2010): Demografischer Wandel als Herausforderung für die Sicherung und Entwicklung einer kosten- und ressourceneffizienten Abwasserinfrastruktur. Dessau-Roßlau. = UBA-Texte 36/2010.

Kerber, H.; Lux, A. (2016): Zum Umgang mit Nachhaltigkeitsrisiken. Erste Ansätze für Maßnahmen aus dem Projekt NaCoSi. Frankfurt am Main. = ISOE-Materialien Soziale Ökologie 46.

Kerber, H.; Schramm, E.; Winker, M. (2016): Transformationsrisiken bearbeiten. Umsetzung differenzierter Wasserinfrastruktursysteme durch Kooperation. Berlin. = netWORKS-Paper 28.
Kersten, J.; Neu, C.; Vogel, B. (2012): Demografie und Demokratie. Zur Politisierung des Wohlfahrtsstaates. Hamburg.

Köhler, H.; Meyer, C. C. (2006): Abwasserabgabengesetz. Kommentar. München.

Kopp-Assenmacher, S. (2015): Wasserrecht und Abfallrecht. In: Lecher, K.; Lühr, H.-P.; Zanke, U. C. E. (Hrsg.): Taschenbuch der Wasserwirtschaft. Grundlagen - Planungen-Maßnahmen. Wiesbaden, 459-492. doi: 10.1007/978-3-8348-8216-5

Libbe, J.; Schramm, E.; Winker, M.; Deffner, J. (2017): Integrierte Infrastrukturplanung. In: Winker, M.; Trapp, J. H.; Libbe, J.; Schramm, E. (Hrsg.): Wasserinfrastruktur: Den Wandel gestalten. Technische Varianten, räumliche Potenziale, institutionelle Spielräume. Berlin, 81-90. = Edition Difu 16.

Libbe, J.; Trapp, J. H.; Winker, M. (2017): Derzeitige Ausgangslage. In: Winker, M.; Trapp, J. H.; Libbe, J.; Schramm, E. (Hrsg.): Wasserinfrastruktur: Den Wandel gestalten. Technische Varianten, räumliche Potenziale, institutionelle Spielräume. Berlin, 29-33. = Edition Difu 16.

Maier, K.; Wolf, M.; Londong, J. (2017): Die Neuorganisation der ländlichen Abwasserentsorgung. In: Wasserwirtschaft Wassertechnik 67, 7-8, 34-37.

Nisipeanu, P. (1991): Abwasserrecht. München. = Praxis des Verwaltungsrechts 4.

Peine, F.-J. (2008): Allgemeines Verwaltungsrecht. Heidelberg.

QLA - Gesellschaft für Qualitätssicherung Landbauliche Abfallverwertung (2017): Qualitäts- und Prüfbestimmungen für Klärschlämme. Rheinbach. http://www.qla.de/sites/default/ files/Dokumente/QP_Klaerschlamm_November_2017.pdf (07.01.2020).

Rost, G.; Maier, K.; Böhm, M.; Londong, J. (2015): Auswirkungen eines technischen Paradigmenwechsels auf die wasserwirtschaftliche Organisation in strukturschwachen ländlichen Räumen. In: Raumforschung und Raumordnung 73, 5, 343-356. doi: 10.007/s13147-015-0362-7

Sartorius, $\quad$ C.; $\quad H i l l e n b r a n d, \quad$ T. $\quad$ (2008): Abwasserentsorgungstechnologie im Elbegebiet - Bestand und Entwicklung. In: Korrespondenz Abwasser Abfall 55, 4, 381-386.

Schmidt, R. (2007): Allgemeines Verwaltungsrecht. Grasberg bei Bremen.

Schön, S.; Kruse, S.; Meister, M.; Nölting, B.; Ohlhorst, D. (2007): Handbuch Konstellationsanalyse. Ein interdisziplinäres Brückenkonzept für die Nachhaltigkeits-, Technik- und Innovationsforschung. München.

Schramm, E.; Kerber, H. (2017): Transformation von Wasserinfrastrukturen: Fördernde und hemmende Faktoren aus Sicht zentraler Akteure. In: Winker, M.; Trapp, J. H.; Libbe, J.; Schramm, E. (Hrsg.): Wasserinfrastruktur: Den Wandel gestalten. Technische Varianten, räumliche Potenziale, institutionelle Spielräume. Berlin, 57-70. = Edition Difu 16.

Schramm, E.; Kerber, H.; Trapp, J. H.; Zimmermann, M.; Winker, M. (2018): Novel urban water systems in Germany: governance structures to encourage transformation. In: Urban Water Journal 15, 6, 534-543. doi: 10.1080/1573062X.2017.1293694

Schulz, P.-M. (2018): Rechtlicher Rahmen für die Verwertung von Stoffen aus neuartigen Sanitärsystemen. In: Zeitschrift für Deutsches und Europäisches Wasser-, Abwasser- und Bodenschutzrecht 7, 4, 204-214. 
Schulz, P.-M. (2019): Rechtliche Ausgestaltung der Einführung von neuartigen Sanitärsystemen bei öffentlichenAbwasseranlagen. In: Korrespondenz Abwasser Abfall 66, 1, 42-50.

Spoth, K.; Schmidt, J.; Arnold, U. (2012): Landwirte und Konsumenten - wie weit geht die Akzeptanz der Nutzung von Urin und Urinprodukten? In: DWA - Deutsche Vereinigung für Wasserwirtschaft, Abwasser und Abfall (Hrsg.): Neue Wasserinfrastrukturkonzepte in der Stadtplanung. Hennef, 99-112.

Starck, C. (1991): Das Verwaltungsermessen und dessen gerichtliche Kontrolle. In Franßen, E.; Redeker, K.; Schlichter, O.; Wilke, D. (Hrsg.): Bürger, Richter, Staat. Festschrift für Horst Sendler zum Abschied aus seinem Amt. München, 167181.

Tauchmann, H.; Hafkesbrink, J.; Nisipeanu, P.; Thomzik, M.; Bäumer, A.; Brauer, A.; Clausen, H.; Drouet, D.; Engel, D.; Körkemeyer, K.; Rothgang, M.; Schroll, M. (2006): Innovationen für eine nachhaltige Wasserwirtschaft. Einflussfaktoren und Handlungsbedarf. Heidelberg. doi: 10.1007/3-7908-1685-X

TLUG - Thüringer Landesanstalt für Umwelt und Geologie (2009): Abwasserbeseitigungspflichtige im Freistaat Thüringen. Karte der Abwasserzweckverbände, Körperschaften mit Zweckvereinbarungen und Eigenentsorger. Jena. = Schriftenreihe der Thüringer Landesanstalt für Umwelt und Geologie 87.

TMLFUN - Thüringer Ministerium für Landwirtschaft, Forsten, Umwelt und Naturschutz (2012): Grundsätze zur Aufstellung von Abwasserbeseitigungskonzepten (ABK) im Freistaat Thüringen. Erfurt.

TMUEN - Thüringer Ministerium für Umwelt, Energie und Naturschutz (2016): Thüringer Landesprogramm Gewässerschutz 2016-2021. Erfurt.

TMUEN - Thüringer Ministerium für Umwelt, Energie und Naturschutz (2018a): Richtlinie für die Förderung von Vorhaben der Abwasserentsorgung im Freistaat Thüringen des Thüringer Ministeriums für Umwelt, Energie und Naturschutz. Erfurt.

TMUEN - Thüringer Ministerium für Umwelt, Energie und Naturschutz (2018b): Richtlinie zur Förderung von Kleinkläranlagen im Freistaat Thüringen des Thüringer Ministeriums für Umwelt, Energie und Naturschutz. Erfurt.

TMUEN - Thüringer Ministerium für Umwelt, Energie und Naturschutz (2018c): Richtlinie für die Förderung von Aus- und Fortbildung und innovativen Maßnahmen im Abwasserbereich aus dem Aufkommen aus der Abwasserabgabe. Erfurt.

Winker, M.; Brüning, H.; Meyer, C.; Schramm, E.; Stieß, I. (2019): Die Akzeptanz ist da. Repräsentative Studie zur Grauwassernutzung in Norderstedt. In: fbr-wasserspiegel 24, 3, 12-17.

WSWU - Weiterbildendes Studium Wasser und Umwelt, BauhausUniversität Weimar (2015): Neuartige Sanitärsysteme. Begriffe, Stoffströme, Behandlung von Schwarz-, Braun-, Gelb-, Grauund Regenwasser, Stoffliche Nutzung. Weimar.

Zimmermann, M.; Felmeden, J.; Michel, B. (2018): Integrated Assessment of Novel Urban Water Infrastructures in Frankfurt am Main and Hamburg, Germany. In: Water 10, 2, 211. doi: 10.3390/w10020211 